\title{
A Study of Spectrum Sharing Technology Based on 230MHz Private Spectrum of Electric Power System
}

\author{
Yidong Yuan ${ }^{1, a}$, Dongyan Zhao ${ }^{1}$, Guangyu $\mathrm{Wu}^{2, \mathrm{~b}}$, Gang $\mathrm{Xie}^{2}$ \\ ${ }^{1}$ Beijing NARI Smartchip Microelectronics Company Limited, China. \\ ${ }^{2}$ Beijing University of Posts and Telecommunications, China. \\ aemail: yuanyidong@sgitg.sgcc.com.cn, bemail: gywu@bupt.edu.cn
}

\section{Keywords: Private wireless network; Spectrum sharing; OFDM; Power allocation}

\begin{abstract}
Private wireless network communications technology is a key technology of the smart grid, but the scarce spectrum resources greatly restrict the development of the private wireless network communications technology. At present, the $230 \mathrm{MHz}$ spectrum can only support a low transfer rate, makes it hard for some emerging business requirements, also restrict the development of traditional business. To solve the emergence of the cognitive radio spectrum resources, realize the dynamic management of spectrum and improve the utilization rate of spectrum, the spectrum sharing technology was proposed to effectively solve the spectrum scarcity. In order to further promote the implementation of the smart grid, this paper puts forward a kind of transmission scheme based on the technology of OFDM and power allocation, and then puts forward a new spectrum planning scheme based on spectrum sharing. The simulation results show that the scheme can effectively increase the system capacity, give a planning scheme of $230 \mathrm{MHz}$ spectrum to the State Radio Regulatory Commission of the People's Republic of China(SRRC), and provides a good reference to promote the development of smart grid provides.
\end{abstract}

\section{Introduction}

With the development of economy and society, network scale is growing stronger. The traditional communications business with low rate can't meet all the requirements for the smart grid, followed by the rise of new business requirements, such as video monitoring, emergency repair and so on. But different business requires vary communication speed or communication quality, which leads to the demand of transmission bandwidth different. In order to support both the rate and quality requirements of different business, a technology with higher spectrum efficiency and flexible allocation of bandwidth is needed. Spectrum sharing based on Cognitive Radio(CR) is an effective technology to solve the problem of spectrum scarcity. Spectrum sharing's main goal is to maximize the spectrum utilization efficiency while keeping fairness between shared users. At present, the research of spectrum sharing in CR mainly based on the spectrum sharing pool strategy[1]. The basic idea of the shared pool is merging part of the spectrum allocated to different business spectrum into a public pool, and then divided the entire spectrum pool into several sub-channel. Therefore, the channel is the basic unit of spectrum allocation. The spectrum sharing problem can be converted into channel allocation problem, to maximize the channel utilization efficiency, and consider interference minimizing and the fairness of accessing at the same time.

As an extension of the backbone network of power system, private wireless network of power system is the main communication infrastructure and the important guarantee to realize the smart grid, automatic power distribution, the charging infrastructure of electric car, the acquisition of user's states information[1]. The development of smart grid put forward higher requirements for the network structure of wireless private network, coverage, bandwidth requirements, application of technology and business management[2].

$230 \mathrm{MHz}$ private spectrum of power system is specifically allocated by the SRRC for energy, hydraulic, geological, and other industries of special spectrum. Dedicated frequency can ensure that the user to deploy a communication private network without any restrictions. The network has a completely independent of management, scheduling, use, distribution, and other functions, and can 
unified planning and construction for municipal network, provincial network and the national network. Due to the scarcity of frequency resources, it is very necessary to research the design of $230 \mathrm{MHz}$ private spectrum of power system communication technology, save frequency resources, improve system capacity and improve the real-time performance and reliability of communication.

At present, the transmission scheme based on $230 \mathrm{MHz}$ private spectrum of power system can only support a low transfer rate, in order to promote the development of smart grid, we must improve the efficiency of $230 \mathrm{MHz}$ frequency spectrum, to support a higher rate and quality requirements of the business.

Orthogonal frequency division multiplexing (OFDM) technology can effectively improve the spectrum's efficiency, increase the system capacity, as well as reducing the multipath interference. At the same time, the actual channel is divided into several sub-channel in OFDM, one of the benefits is that transmission power can be allocation flexibly according to the actual situation of each channel, which will improve the system capacity[3]. In order to improve the spectrum efficiency of $230 \mathrm{MHz}$ private spectrum of power system, we put forward a transmission scheme based on OFDM and power allocation technology.

Due to the multipath effect, the different frequency has different decay, which means that different channel has different channel quality. In order to further improve the system capacity, we proposed a frequency planning scheme based on spectrum sharing. We can improve the system capacity by choosing the better channels in given spectrum to transmission. This scheme gives an effective reference for SRRC to improve the planning scheme of $230 \mathrm{MHz}$ spectrum and promote the development of smart grid.

\section{Analysis of the Private Wireless Network}

Not long ago, the first TD-LTE 230MHz power wireless broadband communication systems was built in a county named HaiYanin, ZheJiang province. It would provide special wireless signal channel for the smart grid. It is a major breakthrough in the smart grid communication technology.

According to the report, with the advancement of construction of the HaiYan's smart grid, the way by renting the public resources have been unable to meet the development of smart grid with new business for bandwidth, real-time and the requirements of the safety and reliability of data transmission. Besides, they have to pay the high rent and technical services each year. Therefore, the solutions of this problem have become a top priority for the construction of smart grid. The private wireless network of power system as a kind of effective solution, has become one of the key technologies to realize the smart grid.

The power enterprise puts its main basic business into three aspects: information collection, distributed automation and emergency repair, maintenance and visualization of mobile assets management. With the development of the smart grid, the characteristics of informatization, digitization, automation and interaction has become more stand out. So we can draw the conclusion that, in the near future, the amount of data information collection will enter a rapid growth phase, while the distributed automation will have higher real-time and quality requirements. Emergency repair, maintenance and visualization of mobile assets management business involves video monitoring implementation, so it transmits data, voice but also image and video, which gets a highest demand for bandwidth among the three services [4].

Known from the above analysis, with the development of smart grid, the traditional digital radio spectrum utilization rate is too low to support some of the emerging business requirements of transmission rate, also cannot meet the needs of intelligent with electric business growing. At the same time, with the continuous development of smart grid, predictably, more and more diversified demand will be put forward. In order to make the best of $230 \mathrm{MHz}$ scarce spectrum resources, improve the spectrum transmission rate and spectrum efficiency is necessary. 


\section{Design for OFDM Scheme Used in the Private Wireless Network of Power System}

Wireless channel is affected by multipath transmission, which can make the frequency selective fading. In the face of bad wireless communication environment and the scarce spectrum resources, it is necessary to design good fading resistance and high frequency band utilization channel. In general serial data system, each data symbol fully occupies the channel available bandwidth. Due to the sudden Rayleigh fading, it will cause the destroying and losing of a few bits during signal fading. The parallel system can reduce this phenomenon in serial transmission.

The basic principle of orthogonal frequency division multiplexing (OFDM) is change the high speed data flow from serial to parallel, and assigned them to the $\mathrm{N}$ sub-channel which transmission rate is relatively low. Since the symbol period of each sub-channel will be relatively increased, it is possible to effectively reduce the impact of the transmission system caused by the radio channel multipath delay. And we can also insert guard interval between the OFDM signals. Since the guard interval is greater than the maximum delay of the wireless channel, we can eliminate inter symbol interference caused by the multipath time delay. Generally, we use a cyclic prefix as guard interval to avoid inter channel interference (ICI) caused by multipath.

Assuming the original serial signal period is $t_{s}$, the parallel signal period is $T_{s}$, since we change the signal from serial to parallel, each period of the signal changed into $N$ times to the original signal period, that is $T_{s}=N t_{s}$, the rate of the sub-channel changed into $1 / T_{s}=1 / N t_{s}$, the $\mathrm{N}$ sub-channels are:

$$
f_{n}=f_{0}+n \Delta f \quad n=0,1,2, \ldots, N-1
$$

Where $\Delta f=1 / T_{s}=1 / N t_{s}$, when $f_{0} \gg \Delta f$, each subcarrier is orthogonal, that meet:

$$
\frac{1}{T_{S}} \int_{0}^{T_{S}} \exp \left(j 2 \pi f_{i} t\right) \exp \left(-j 2 \pi f_{j} t\right) d t=0
$$

Among them $f_{j}-f_{i}=\frac{k}{T_{S}}(k=1,2,3 \ldots)$, each branch can be modulated by PSK, QAM and other digital modulation techniques. Sum the N modulating signals, the OFDM signal can be obtained:

$$
d(t)=\sum_{n=0}^{N-1} d(n) \exp \left[j 2 \pi\left(f_{0}+\frac{n}{T}\right) t\right]
$$

At the receiving end, the received signal enter into $\mathrm{N}$ parallel branches at the same time, through coherent demodulation respectively, we can restore the data of parallel branch. The demodulation and integration to the $\mathrm{m}^{\text {th }}$ sub-carrier is showed that:

$$
\begin{aligned}
\mathrm{d}^{\prime}(\mathrm{m}) & =\frac{1}{\mathrm{~T}_{\mathrm{S}}} \int_{0}^{\mathrm{T}_{\mathrm{S}}} \mathrm{D}(\mathrm{t}) \exp \left[-\mathrm{j} 2 \pi\left(\mathrm{f}_{0}+\frac{\mathrm{m}}{\mathrm{T}}\right) \mathrm{t}\right] d t \\
& =\frac{1}{T_{S}} \sum_{\mathrm{n}=0}^{\mathrm{N}-1} d(n) \int_{0}^{\mathrm{T}_{\mathrm{S}}} \exp \left(j 2 \pi \frac{n-m}{T} t\right) d t=d(n)
\end{aligned}
$$

As can be seen from the above equation, the demodulation to the $\mathrm{m}^{\text {th }}$ sub-carrier can recover the original symbol, for other sub-carriers, $(\mathrm{nm}) / \mathrm{T}$ produces an integer multiple of the cycle, so the integration result is zero.

In OFDM system design, it is necessary to take into account compromise between various system requirements, these requirements are often contradictory. Usually there are three main system parameters needs to be addressed: system bandwidth $\mathrm{W}$, business transfer rate $\mathrm{R}$ and multipath time delay expend $\tau$.

In the $230 \mathrm{MHz}$ spectrum planned by the Ministry of Industry and Information SRRC, sub-carrier spacing to $25 \mathrm{KHZ}$, including 40 points of the electric private wireless network, namely 40 channels, a total of $1 \mathrm{M}$ bandwidth. Therefore, OFDM signal bandwidth applied in the private wireless network of power system should be less than or equal to $1 \mathrm{M}$, here we design of OFDM signal bandwidth $\mathrm{W}$ is $1 \mathrm{M}$.

OFDM signal between sub-carriers must meet the orthogonal conditions, due to the $230 \mathrm{MHz}$ 
private wireless network is a discrete distribution in spectrum, which leads to discontinuity between OFDM subcarriers, it is difficult to design the OFDM system parameter. In order to ensure orthogonality, we design subcarrier spacing $\Delta \mathrm{f}$ is $25 \mathrm{KHz}$, exactly half of the channel spacing planned by the SRRC, so even if the subcarrier is a discrete distribution, it can still ensure that the difference between the two sub-carriers in times of $\Delta \mathrm{f}$, means ensures orthogonality between subcarriers, while ensuring each subcarrier bandwidth $25 \mathrm{KHz}$, will not exceed the channel bandwidth.

According to the above design, the OFDM signal subcarrier number $\mathrm{N}$ should be $\frac{\mathrm{w}}{\Delta \mathrm{f}}=40$, integral duration $_{\text {OFDM }}=\frac{1}{\Delta \mathrm{f}}=40$ us.

Then we determine the protection time $\mathrm{T}_{\mathrm{G}}$. Multipath time delay directly decides the size of the protection time. As an important design criterion, protect time shall be at least $2 \sim 4$ times the RMS(Root Mean Square) of multipath time delay, that is $T_{G} \geq(2 \sim 4) \tau_{r m s}$. In the power system, since most mobile terminals are fixedly mounted, so $\tau_{\mathrm{rms}}$ small, that is $\mathrm{T}_{\mathrm{G}} \ll \mathrm{T}_{\mathrm{OFDM}}$, it means that the design requirements of $\mathrm{T}_{\mathrm{G}}$ are more lenient. $\mathrm{T}_{\mathrm{OFDM}}$ is general guaranteed at least 5 times $\mathrm{T}_{\mathrm{G}}$ in industry, so we design $T_{G}=8 \mathrm{us}$. So the OFDM symbol duration time $T_{s}=T_{G}+\mathrm{T}_{\mathrm{OFDM}}=48 \mathrm{us}$.

Because the industry generally uses the Fast Fourier Transform FFT to implement OFDM signal, it requires the number of sampling points to be an integer power of 2.The number of sampling points can be designed to 64, so we need to oversample to the OFDM signal, which adds 24 zeros carrier. Because zero carriers no carry the information, it will not cause the expanding of system bandwidth.

An additional requirement of the system design is to ensure the sample number is integer during the Symbol period $T_{s}$ and the processing time of FFT/IFFT. Based on the above analysis, we should ensure the sample number is precisely 64 sample values in the processing time of FFT/IFFT. Choose the sampling rate is $\frac{64}{40 \mathrm{us}}=1.6 \mathrm{MHz}$ can satisfy this requirement. But the sampling rate can't get an integer sample value in 48 us symbol period $(1.6 \mathrm{MHz}$ x $48 \mathrm{us}=76.8)$. The solution is slightly reduces the requirement to some of the parameter, to meet the integer constraints. Base on the above analysis, electric system has loose requirement on the protection time $T_{G}$, so we reduce the $\mathrm{T}_{\mathrm{G}}$ to $5 \mathrm{us}$, then the sample number of each OFDM symbol is $1.6 \mathrm{MHz} \times 45 \mathrm{us}=72$, which can satisfy the integer constraints, and do not need to change other parameters at the same time.

\section{Power Allocation Algorithm Design}

The Shannon theorem is pointed out that, in theory, as long as the actual transfer rate is lower than the channel capacity, it is possible to transmit information with arbitrarily small error rate in the channel. Therefore, the channel capacity is the maximum transmission limit to realize reliable communication. Orthogonal frequency division multiplexing (OFDM) divide the actual into several sub-channels, one of the benefits is that we can allocate the power flexibly according to the actual situation of each channel, which can improve the system capacity.

In this paper, the research of OFDM system is composed of a base station and multiple users, the subcarrier number is $\mathrm{N}$. The total power of the system is under certain constraints, that is it need to meet:

$$
\sum_{i=1}^{N} P\left(f_{i}\right) \leq P_{T}
$$

$P\left(f_{i}\right)$ denote the power allocated to each sub-channel, $P_{T}$ denote the total power of the system. It is common sense that the power allocated to sub-channel $P\left(f_{i}\right)$ need to satisfy that:

$$
P\left(f_{i}\right) \geq 0
$$

In conclusion, the optimal object of the OFDM system researched in this paper is to maximize the system capacity under the two power constrains, that is: 


\section{$\max \mathrm{C}$}

$$
\text { s.t. }\left\{\begin{array}{c}
\sum_{n=1}^{N} P\left(f_{i}\right) \leq P_{T}, \\
P\left(f_{i}\right) \geq 0
\end{array}\right.
$$

The above optimization problem is an optimization problem with constraints. Normally, for equality constraint, we can use the Lagrange multiplier method to solve, for inequality constraints, use the KKT(Karush-Kuhn-Tucker) condition to solve. The following is the solving process of Lagrange multiplier method.

By dividing the available channel bandwidth into several narrow sub-channels, the transmission performance of each channel can be close to the ideal. Assuming $H(f)$ is the transmission function of a channel has $\mathrm{WHz}$ bandwidth. There is additive Gaussian noise which power spectral density is $\mathrm{N}(\mathrm{f})$ in the channel. So we can divide the channel into $\mathrm{N}=\mathrm{W} / \Delta f$ sub-channel, $\Delta f$ is the bandwidth of each sub-channel.They should satisfy the following condition: $H(f)^{2} / N(f)$ inone sub-channel bandwidth is approximately constant, and signal transmit power meet the constraint of (7).

In AWGN channel, the channel capacity can be expressed as:

$$
C=W \log _{2}\left(1+\frac{P_{T}}{W N_{0}}\right)
$$

In multi-carrier system, if $\Delta f$ is small enough, it means that the parameters are constant in the range of sub-channel frequency, then the sub-channel capacity can be expressed as:

$$
\mathrm{C}_{\mathrm{i}}=\Delta \mathrm{f} \log _{2}\left[1+\frac{\Delta \mathrm{fP}\left(\mathrm{f}_{\mathrm{i}}\right) \mathrm{H}\left(\mathrm{f}_{\mathrm{i}}\right)^{2}}{\Delta \mathrm{fN}\left(\mathrm{f}_{\mathrm{i}}\right)}\right]
$$

Therefore, the total channel capacity can be expressed as:

$$
C=\sum_{i=1}^{N} C_{i}=\Delta f \sum_{i=1}^{N} \log _{2}\left[1+\frac{P\left(f_{i}\right) H\left(f_{i}\right)^{2}}{N\left(f_{i}\right)}\right]
$$

If $\Delta f \rightarrow 0, \quad(9)$ can be replaced by following:

$$
C=\int_{W} \log _{2}\left[1+\frac{\Delta f P\left(f_{i}\right) H\left(f_{i}\right)^{2}}{\Delta f N\left(f_{i}\right)}\right] d f
$$

Considering the restricted condition of (7), using calculus of variations change (11) into (12) to maximal the capacity of the channel:

$$
\left\{\int_{W} \log _{2}\left[1+\frac{\Delta f P\left(f_{i}\right) H\left(f_{i}\right)^{2}}{\Delta f N\left(f_{i}\right)}\right]+\lambda P(f)\right\} d f \rightarrow \max
$$

$\lambda$ is a Lagrange multiplier, which value need to meet the restricted condition of (7)。After transformation, the above formula turns into that:

$$
P(f)= \begin{cases}K-\frac{N(f)}{H(f)} & f \in W \\ 0 & f \notin W\end{cases}
$$

The physical meaning of the above formula is substantially: when the SNR $H(f)^{2} / N(f)$ is large, corresponding power of the channel should also be greater, and when the SNR is lower, signal power should be lower too. Figure 1 shows the power distribution of transmission signal. As shown in the figure, the solid curve shows the reciprocal of different SNR for different frequency in the channel bandwidth. The method to maximize channel capacity is similar to the putting water 
(shaded area, $\mathrm{P}_{\mathrm{av}}$ ) into the bowl solid curve represented, then we can obtained $\mathrm{P}(\mathrm{f})$ which can maximal the channel capacity. This is the method that we call water-filling.

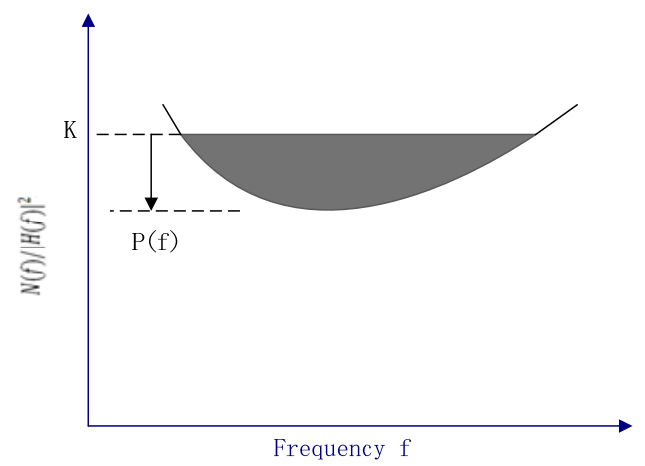

Fig1.The schematic diagram of water-filling

- In practical application, since the optimal water distribution method is a problem that the algorithm complexity is very high, the Lagrange multiplier method is usually used to obtain the suboptimal solution of power allocation. So we used the Lagrange multiplier method in MATLAB simulation in the following part.

In order to further improve the system capacity, based on the basis of the conventional power allocation method mentioned above, this paper proposes a new power allocation scheme by considering the spectrum sharing and using the greedy algorithm.

Due to the time-varying characteristics of the wireless channel, different frequencies have different fading characteristics, that is frequency selective fading. Based on this feature, we use the water-filling power allocation algorithm to improve the system capacity. Now consider the problem of spectrum sharing. Assuming we can use spectrum sharing to deal with all the $12 \mathrm{MHz}$ frequencies in 230MHzspectrum planned by the SRRC, then we can make use of the greedy algorithm to choose the best quality $1 \mathrm{MHz}$ bandwidth to the power allocation. Most of the wireless channel delay spread $\tau_{\max }$ is 5 uscommonly, means coherent bandwidth is $\mathrm{f}=\frac{1}{\tau_{\max }}=250 \mathrm{KHz}, 12 \mathrm{MHz} \gg$ $250 \mathrm{KHz}$. Therefore, predictably, the power allocation scheme based on spectrum sharing, can effectively improve the system capacity. The simulation result is given below.

\section{The Simulation and Result Analysis}

In this part, we gave the simulation of the power allocation of OFDM system used in the Electric Private Wireless Network, and observe its impact on system capacity. Simulation adopted COST207 typical city 6 diameter frequency selective fading channel model, specific parameters are as follows in table 1.

Based on COST207 multipath channel model, adopting the design parameters of OFDM system above in this paper, then we make the simulation to the power allocation of OFDM system used in the $1 \mathrm{MHz}$ private wireless network of power system planned by the SRRC, simulation results are shown in figure 2.

Seen from the simulation results, we can significantly improve the system capacity by using the water-filling power allocation when the signal-to-noise ratio(SNR) is low. But with the increase of signal-to-noise ratio, the improve proportion become less. This is because at any given set of channel realization, when the signal-to-noise ratio tends to infinity, the power allocated to each channel have tended to a same value $\mathrm{K}$ (see equation (13)), then the system capacity tend to a certain value, namely, the capacity of the system using water-filling power allocation and the system using average power allocation reached by the same, resulting in the performance reducing. 
Table 1.A typical urban channel parameters

\begin{tabular}{|c|c|c|c|}
\hline Path & Delay/us & Gain/dB & $\begin{array}{c}\text { Delay } \\
\text { spread/us }\end{array}$ \\
\hline 0 & 0 & -3 & 1.0 \\
\hline 1 & 0.2 & -0 & 1.0 \\
\hline 2 & 0.6 & -2 & 1.0 \\
\hline 3 & 1.6 & -6 & 1.0 \\
\hline 4 & 2.3 & -8 & 1.0 \\
\hline 5 & 5.0 & -10 & 1.0 \\
\hline
\end{tabular}

The simulation result of power allocation based on the spectrum sharing is shown in figure 3 . It can see that the power allocation scheme based on spectrum sharing indeed can effectively improve the system capacity. Obvious, by using the solution, we can greatly improve the spectrum efficiency of the private wireless network of power system, so it can bear a higher rate of business, and effectively promote the development of smart grid. At the same time, the new planning thought, also provides SRRC a valuable reference to improve the planning of the $230 \mathrm{MHz}$ spectrum.

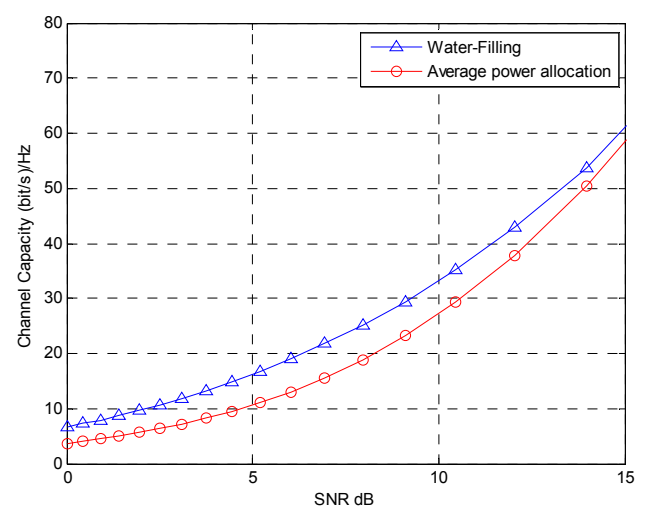

Fig 2.System capacity of two kinds of power allocation algorithm

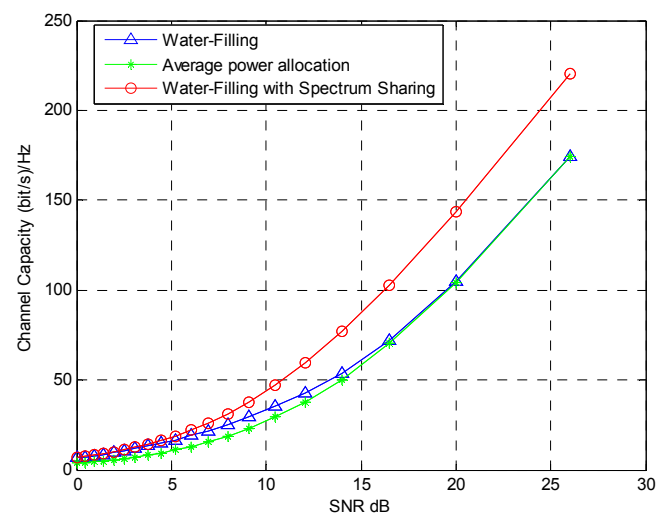

Fig 3.System capacity of the way use spectrum sharing and no use spectrum sharing 


\section{Conclusion}

In order to adapt to the rapid development of the business needs of the smart grid, this paper puts forward an OFDM transmission scheme based on cognitive radio. Aiming at the characteristic of $230 \mathrm{MHz}$ Private Spectrum of Power System, this article analyses and designs the parameters of OFDM system. At the same time, in order to improve the system capacity, we use the water-filling power allocation method to allocate the power, and then make the simulation for it. This article also puts forward a frequency planning scheme based on spectrum sharing, and seen from the simulation result, the scheme can effectively improve the system capacity, providing an effective reference for SRRC to improve the planning scheme of $230 \mathrm{MHz}$ spectrum and promote the development of smart grid.

However, it is obvious that the proposed scheme in this article is based on the ideal condition. So in the practical application, there still are many problems need to be solved. We believe that with the deepening of the research, spectrum sharing technology can play a bigger role in the field of smart grid and communication.

\section{References}

[1] Jinping Cao, Jianming Liu, Shaomin Zhu, YiyingZhang. A wide coverage wireless communication scheme for the intelligent distribution network. Information and Communication Technologies (WICT).pp. 999-1003, 2012.

[2] Ipakchi, A.; Albuyeh, F., Grid of the future, in Power and Energy Magazine, IEEE , vol.7, no.2, pp.52-62, March-April 2009.

[3] Cheong Yui Wong, Cheng, R.S., Lataief, K.B., Murch, R.D.. Multiuser OFDM with adaptive subcarrier, bit, and power allocation, Selected Areas in Communications, IEEE Journal on, Volume: 17 Issue: 10 , Oct. 1999 , pp. 1747.1758

[4] Tang,G.Q., Smart grid management \&visualization: Smart Power Management System. Emerging Technologies for a Smarter World (CEWIT).pp.1-6, 2011.

[5] Tore Ulversøy. Software Defined Radio: Challenges and Opportunities[J]. IEEE COMMUNICATIONS SURVEYS \&TUTORIALS, VOL. 12, NO.4, FOURTH QUARTER 2010.

[6] Asad A. Abidi, Fellow, IEEE. The Path to the Software-Defined Radio Receiver[J]. IEEE JOURNAL OF SOLID-STATE CIRCUITS, VOL. 42, NO. 5, MAY 2007.

[7] Erik Axell, Geert Leus, Erik G. Larsson, and H. Vincent Poor. Spectrum Sensing for Cognitive Radio. IEEE Signal Processing Magazine. pp. 101-116. MAY 2012.

[8] J. Jang and K. Lee, Transmit power adaptation for multiuser OFDM systems, IEEE Journal on Selected Areas in Communications, vol. 21, no. 2, pp. 171-178, Feb 2003. 